\title{
Single- and double-layer microwave absorbers of cobalt ferrite and graphite composite at gigahertz frequency
}

\begin{abstract}
Microwave absorbers of cobalt ferrite and graphite composite have been fabricated to investigate the broadening of absorption frequency by mixing small and large particles and comparing single- and double-layer composites in order to broaden the absorption frequency and to enhance multireflection and scattering of electromagnetic wave. $\mathrm{CoO}$ and $\mathrm{Fe} 2 \mathrm{O} 3$ precursors were mechanically alloyed and sintered at 800, 900, 1000 and $1100 \circ \mathrm{C}$ in order to synthesise various particle sizes and shapes. A mixed sample of powders sintered at the above mentioned temperatures was also formed. The morphological, phase, and magnetic properties of these samples were studied. Two series of samples have been synthesised whichwere singleand double-layer composites. Single-layer composite consisted of mixed particles, and their thickness parameters were varied. Double-layer composite was also prepared with various particle sizes of $\mathrm{CoFe} 2 \mathrm{O} 4$ as a layer and graphene as another layer.The effect of these layers as matching and absorbing layer was studied. The degree of crystallinity of $\mathrm{CoFe} 2 \mathrm{O} 4$ was increased with increase of sintering temperature. Microstructural study showed the evolution of particle size and shape subjected to sintering temperature. Arrhenius plot showed two stages of growth process with activation energy calculated at 26.85 and $83.091 \mathrm{~kJ} / \mathrm{mol}$. The critical size of single domain of this sample plotted from the coercivity against particle size was 63.44 $\mathrm{nm}$. Single-layer composite with mixed particle size was not able to show good electromagnetic (EM)-wave absorption; however double-layer composite showed good return loss. The mechanism of the EM-wave absorption of double-layer composite was discussed in this paper.
\end{abstract}

Keyword: Cobalt ferrite; Single layer; Double-layer structure; Microwave absorber; Reflection loss (RL) 